\title{
Masquelet technique for management of posttraumatic bone defect in a patient of chronic osteomyelitis of long bone
}

\author{
Lokesh Thakur, Sarvesh Kumar Singh*, Sunny Dua, Devinder Kumar, Manish Sharma
}

Department of Orthopaedics, Rajendra Prasad Government Medical College, Kangra, Tanda, Himachal Pradesh, India

Received: 28 February 2021

Revised: 07 April 2021

Accepted: 08 April 2021

\section{*Correspondence:}

Dr. Sarvesh Kumar Singh,

E-mail: drsarveshsingh9@gmail.com

Copyright: (C) the author(s), publisher and licensee Medip Academy. This is an open-access article distributed under the terms of the Creative Commons Attribution Non-Commercial License, which permits unrestricted non-commercial use, distribution, and reproduction in any medium, provided the original work is properly cited.

\section{ABSTRACT}

Masquelet technique, which is the use of a temporary cement spacer followed by staged bone grafting, is a treatment strategy for reconstruction of large, infected, bone defects. This paper describes a patient treated with this technique to successfully manage postinfective or posttraumatic osseous long bone defect. Sequestrectomy was done and the injured limb was stabilized and aligned with application of external fixator at the time of initial antibiotic cement spacer placement. After 6 weeks of proper antibiotic coverage, all antibiotics were stopped for next 2 weeks. Clinical evaluation and investigations were done to rule out any residual infection. Osseous consolidation was successfully achieved with staged bone grafting and internal stabilization of long bone defect.

Keywords: Masquelet technique, Posttraumatic, Chronic osteomyelitis, Bone defects

\section{INTRODUCTION}

The management of chronic osteomyelitis of long bone with large metadiaphyseal defects along with pathological fracture remains a significant orthopaedic challenge, especially in zones of conflict and humanitarian settings. ${ }^{1}$ The use of the ilizarov technique, vascularized fibular grafts, and acute limb shortening have been used previously to address defects of various lengths. ${ }^{2}$ More recently, the use of an antibiotic cement spacer followed by bone grafting within this space confined by an induced biological membrane has been described as a potential treatment strategy. ${ }^{3,4}$ In 2000, Masquelet et al. reported 35 cases of two-stage bone reconstruction for large, infected, diaphyseal defects. ${ }^{5}$ This paper describes a patient at our institution successfully treated with this technique.

\section{CASE REPORT}

Patient was presented to our institution after posttraumatic event. Historical evidence of radiological and clinical bone infection of distal part of femur right side with knee joint extension (Figure 1). In between of follow up pathological fracture and stabilization as internal fixation of involved site at somewhere other institution was considered (Figure 2 ). On clinical evaluation fever, local bone pain and swelling was noticed. Imaging procedures and laboratory studies were done (Table 1). The patient was evaluated for injury type, location, soft tissue condition, skin ulcer, sinuses and discharge, duration of infection, duration of previous episode of fracture and fixation of same site, limb length discrepancy, and range of motion of knee joint (Table 2). The patient was treated with the induced membrane technique. Implant removal was done. The sequestrum was cleaned and surrounding necrosed and scarred tissues were removed and used for culture and histopathological examination. Bone ends were debrided until signs of punctate bleeding (Paprika sign), and the medullary cavity was reamed. The wound was washed with hydrogen peroxide, dilute povidone-iodine and saline repeatedly. After effective fixation, the bone defect of approximately $12 \mathrm{~cm}$ was filled with antibiotic loaded bone cement at the first stage (Figure 3). We preferred to use $2 \mathrm{~g}$ vancomycin or gentamicin per $40 \mathrm{~g}$ of cement 
prepared. Intravenous injections of sensitive antibiotics were administered for two weeks postoperatively based on the drug sensitivity tests. Between first and second stage weight-bearing was not performed. After 6 weeks, all antibiotics were stopped for next 2 weeks. The situation of infection control was assessed before grafting, including the presence of signs, such as redness, sinus and pus, or abnormal laboratory tests, such as total leucocyte count, erythrocyte sedimentation rate, and C-reactive protein.

The second stage of bone grafting was performed after 8 weeks of first surgery. The amount of bone graft is estimated radiologically before surgery. The site was approached through the previous incision and careful dissection was performed to preserve biomembrane encapsulating the antibiotic loaded cement spacer. The cement spacer was removed and, the biomembrane capsule was irrigated to remove any residual debris. The medullary canal was reamed and wound was washed. The corticocancellous bone grafts taken from ipsilateral iliac crest and fibula was placed to fill the entire defect completely but not overstuffed. The induced biomembrane was closed with absorbable suture. Internal fixation was done with plate and screws construct (Figure 4). Prophylactic antibiotics were administered. Patient underwent followup at 1, 2, 3, 6, 9, 12, and 18 months. We assessed local pain, infection control, range of knee movement, time to bone healing, ability to walk and other complications. Gradual ambulation until callus formation was observed (Figure 5 A-B). After 18 months of second stage surgery radiographic healing as bridging callus on three of four cortices and clinical healing as pain-free full weight bearing walking has been achieved (Figure 6).

Table 1: Clinical Evaluation.

\begin{tabular}{|lc|}
\hline Biological parameter & Observed values \\
\hline Body temperature & $101.3^{0} \mathrm{~F}$ \\
\hline Total leucocyte count & $11000 / \mu \mathrm{l}$ \\
\hline $\begin{array}{l}\text { Erythrocyte } \\
\text { sedimentation rate }\end{array}$ & $34 \mathrm{~mm} / \mathrm{hour}$ \\
\hline C-reactive protein & $>12<24$ (positive) \\
\hline
\end{tabular}

Table 2: Patient Demographics.

\begin{tabular}{|ll|}
\hline Sex/Age (y) & F / 11 \\
\hline Injury type & Closed \\
\hline Location & Distal femur right \\
\hline Skin condition & $\begin{array}{l}\text { Healed scar mark over } \\
\text { right knee }\end{array}$ \\
\hline Skin ulcer & No \\
\hline Sinuses and discharge & No \\
\hline Duration of infection & 10 months \\
\hline $\begin{array}{l}\text { Duration of previous } \\
\text { fracture and fixation }\end{array}$ & 8 months \\
\hline Limb length discrepancy & 5 cm \\
\hline $\begin{array}{l}\text { Range of motion of knee } \\
\text { joint }\end{array}$ & Painful and restricted \\
\hline
\end{tabular}

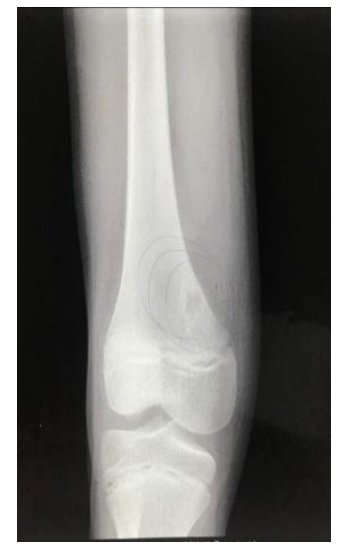

Figure 1: Radiograph ap view, demonstrating well defined lytic lesion in the metaphyseal region of distal femur.

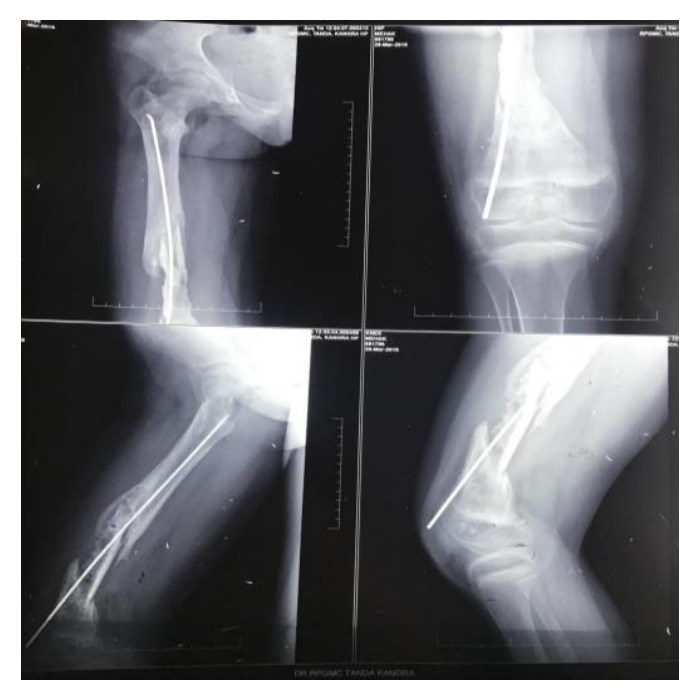

Figure 2: Radiograph ap/lateral view, demonstrating large sequestrum, non-structural, sclerotic, expanded involucrum with bone defect (with implant in situ).

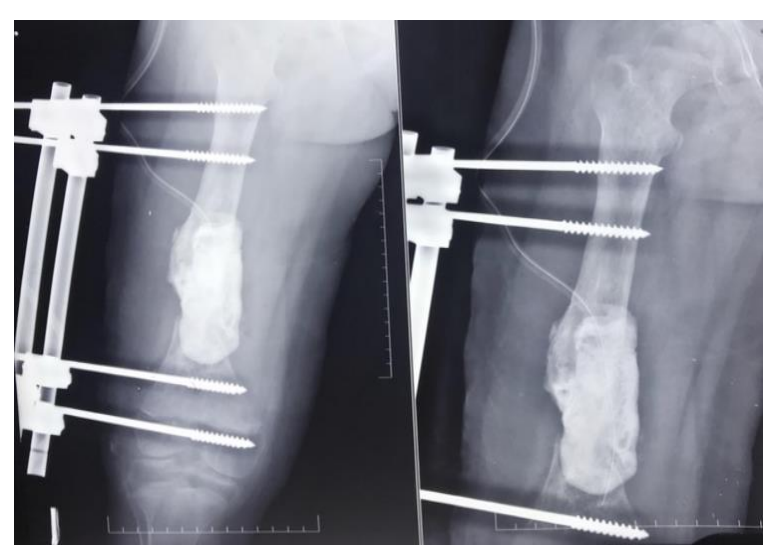

Figure 3: AP and Lateral radiographs showing fixation with external fixator and placement of antibiotic cement spacer into bone defect after adequate debridement. 


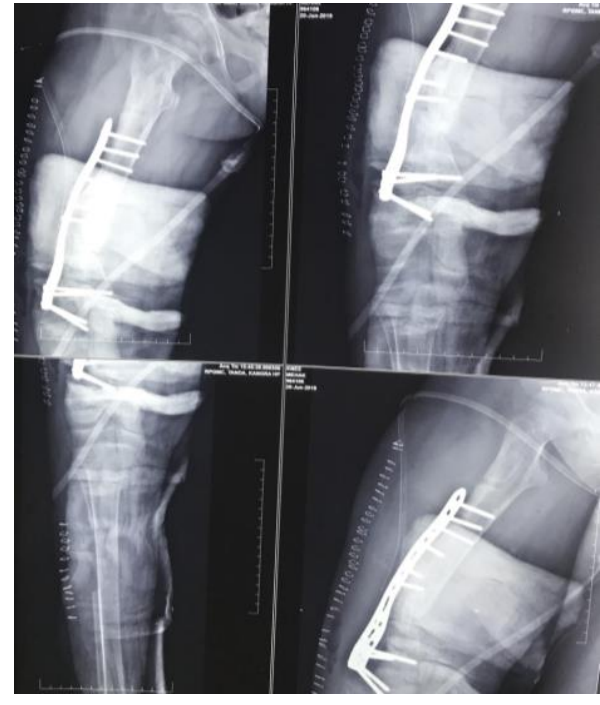

Figure 4: Immediate postoperative, AP and lateral radiographs, showed the antibiotic cement spacer being removed and the defect filled with corticocancellous autograft harvested from ipsilateral iliac crest and fibula. Internal fixation was done with plate and screws.

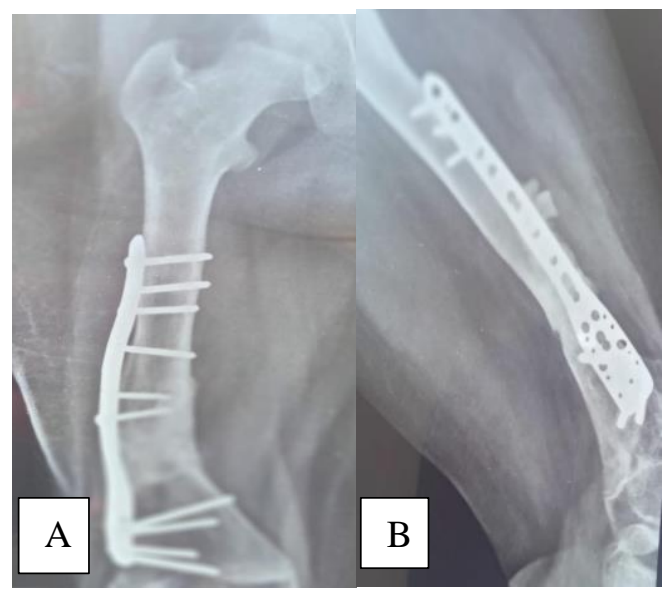

Figure 5: (A, B) AP and lateral radiographs, at 9 months of follow up showing osseous consolidation.

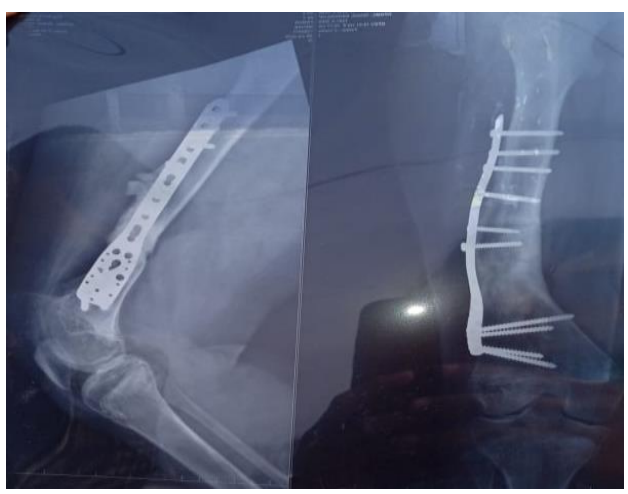

Figure 6: AP and lateral radiographs, at 18 months of follow up showing osseous consolidation.

\section{DISCUSSION}

The advantages of this method are that the induced membrane not only contains the bone graft and prevent its resorption at the early stages; but it also plays an important role in revasularisation, bone formation and consolidation throughout the regeneration process. ${ }^{6}$ In the current report, we describe an extension of this technique with cortical and cancellous bone autograft to achieve anatomic reconstruction of a large bone defect after removal of large sequestrum in a 11-year-old child.

Masquelet and Begue proposed that this membrane prevents graft resorption and improves vascularity and corticaliztion. It has been described that, after the initial placement of the antibiotic impregnated spacer, an interval of 4 to 5 weeks is needed for development and maturation of a biologically active membrane that is suitable for grafting. The spacer also maintains the defect and inhibits fibrous ingrowth. ${ }^{5}$ Viateau et al. studied this technique in a sheep model and found that the membrane alone was inadequate to heal a large bone defect; but when autologous bone graft was palced within the membrane, all the defects went on to heal. ${ }^{7}$ Pelissier et al. reported that the induced membranes secrete growth factors including vascular and osteoinductive factors and could stimulate bone regeneration. ${ }^{8}$

Biau et al. used both iliac crest corticocancellous autograft and a medial tibial cortical strut autograft to fill their large defect. ${ }^{9}$ Use of cancellous autograft from the femoral canal has also been described, and evidence exists to show that levels of many growth factors (fibroblast growth factor- $\alpha$, Platelet derived growth factor, insulin-like growth factor 1, TGF-1, and BMP-2) in femoral cancellous bone are present in higher concentrations than they are in iliac crest and platelet preparations. ${ }^{10}$

Chronic osteomyelitis is a serious problem for orthopedists given the lack of a generally accepted method for treatment. Classic techniques include ilizarov technique, one stage bone grafting and vascularized fibula graft. These techniques are often associated with high complications or long-term recurrence rates. ${ }^{11} \mathrm{We}$ applied the induced membrane technique for the treatment of chronic osteomyelitis. The process involves a series of measures, including radical debridement and removal of necrotic tissue, elimination of dead space, drainage, and stable fixation for infection control. Then, a membrane is formed by implantation of a PMMA cement, and grafting the induced membrane approximately 8 weeks later. The induced biomembrane and corticocancellous autograft rapidly promotes bone defect repair. ${ }^{12}$

In general terms, the vascularized fibular autograft and the Ilizarov bone transfer technique are still the most commonly used methods for reconstruction of large bone defects. ${ }^{13,14}$ Despite their advantages as bone regeneration methods, they are also associated with complications and significant drawbacks. Such disadvantages include the 
considerable donor site morbidity, the demanding microsurgical technique and prolonged operative time, as well as risk of the fracture or inadequate hypertrophy of the graft for the use of vascularized fibula autografts. For distraction osteogenesis, the risk of infection and especially of septic arthritis when used closed to a joint, and the prolonged time required for distraction and consolidation of the regenerate, often dictate the need for an alternative method of bone reconstruction. Therefore, the Masquelet technique as well as other techniques for restoration of large bone defects, such as intramedullary lengthening devices, the use of cylindrical metallic or titanium mesh cages, and the monorail method for segment bone transport, can be used as alternative method for certain cases. ${ }^{15-18}$

Key factors predicting the outcome of reconstruction rate are the presence of infection or vascular deficiency of the extremity, as they are associated with high complication rate (delayed union, non-union, or vascular thrombosis) and a poor functional result. ${ }^{19}$

\section{CONCLUSION}

In general, the key for a good outcome is to understand the overall concept of the induced membrane technique: The pseudo-synovial membrane formed around the cement spacer (as a foreign body reaction- first stage) acts as a chamber around the bony defect to contain the bone graft and stimulate bone regeneration (second stage).

Funding: No funding sources Conflict of interest: None declared

Ethical approval: Not required

\section{REFERENCES}

1. Morelli I, Drago L, George DA, Gallazzi E, Scarponi S, Romanò CL. Masquelet technique: myth or reality? A systematic review and meta-analysis. Injury. 2016;47 S6:68-76.

2. Wong TM, Lau TW, Li X, Fang C, Yeung K, Leung F. Masquelet technique for treatment of posttraumatic bone defects. Scient World J. 2014 Feb 6;2014:710302.

3. Masquelet AC. Muscle reconstruction in reconstructive surgery: soft tissue repair and long bone reconstruction. Langenbecks Arch Surg. 2003;388(5):344-6.

4. Masquelet AC, Begue T. The Concept of Induced Membrane for Reconstruction of Long Bone Defects. Orthoped Clin North Ame. 2010;41(1):27-7.

5. Masquelet AC, Fitoussi F, Begue T, Muller GP. Reconstruction of the long bones by the induced membrane and spongy autograft. Ann Chir Plast Esthet. 2000;45(3):346-53.

6. Giannoudis PV, Faour O, Goff T, Kanakaris N, Dimitriou R. Masquelet technique for the treatment of bone defects: tips-tricks and future directions. Injury. 2011;42(6):591-8.

7. Viateau V, Guillemin G, Calando Y, Logeart D, Oudina K, Sedel L, Hannouche D, et al. Induction of a barrier membrane to facilitate reconstruction of massive segmental diaphyseal bone defects: an ovine model. Vet Surg. 2006;35(5):445-52.

8. Pelissier P, Masquelet AC, Bareille R, Pelissier SM, Amedee J. Induced membranes secrete growth factors including vascular and osteoinductive factors and could stimulate bone regeneration. J Orthop Res. 2004;22(1):73-9.

9. Biau DJ, S. Pannier AC, Masquelet, Glorion C. Case report: reconstruction of a $16-\mathrm{cm}$ diaphyseal defect after Ewing's resection in a child. Clinic Orthopaed Related Res. 2009;467(2):572-7.

10. Schmidmaier G, Herrmann S, Green J, Weber T, Scharfenberger A, Haas NP, et al. Quantitative assessment of growth factors in reaming aspirate, iliac crest, and platelet preparation. Bone. 2006;39(5):1156-63.

11. Dendrinos GK, Kontos S, Lyritsis E. Use of the Ilizarov technique for treatment of non-union of the tibia associated with infection. J Bone Joint Surg Am. 1995;77(6):835-46.

12. Wang $X$, Luo F, Huang $K$ et al. Induced membrane technique for the treatment of bone defects due to post-traumatic osteomyelitis. Bone Joint Res. 2016;5(3):101-5.

13. Pederson WC, Person DW. Long bone reconstruction with vascularized bone grafts. Orthop Clin North Am. 2007;38(1):23-35.

14. Aronson J. Limb-lengthening, Skeletal reconstruction, and bone transport with the Ilizarov method. J Bone Joint Surg Am. 1997;79(8):1234-58.

15. Cole JD, Justin $\mathrm{D}$, Kasparis $\mathrm{T}$ et al. The intramedullary skeletal kinetic distractor (ISKD): First clinical results of a new intramedullary nail for lengthening of the femur and tibia. Injury 2001;32(Suppl. 4):129-39.

16. Bullens PH, Schreuder BHW, Malefijt WMC et al. Is an impacted morselized graft in a cage an alternative for reconstructing segmental diaphyseal defects? Clin Orthop Relat Res. 2009;467(3):783-91.

17. Ostermann PA, Hasse N, Rubberdt A et al. Management of a long segmental defect at the proximal meta-diaphyseal junction of the tibia using a cylindrical titanium mesh cage. J Orthop Trauma 2002;16(8):597-601.

18. Raschke M, Oedekoven G, Ficke J, Claudi BF. The monorail method for segment bone transport. Injury1993;24(S2);54-61.

19. Pelissier P, Boireau P, Martin D, Baudet J. Bone reconstruction of the lower extremity: complications and outcomes. Plast Reconstr Surg. 2003;111(7):2223-9.

Cite this article as: Thakur L, Singh SK, Dua S, Kumar D, Sharma M. Masquelet technique for management of posttraumatic bone defect in a patient of chronic osteomyelitis of long bone. Int $\mathrm{J}$ Res Orthop 2021;7:691-4. 